\title{
Spontaneous interlayer superfluidity in bilayer systems of cold polar molecules
}

\author{
Roman M. Lutchyn ${ }^{1,2 *}$, Enrico Rossi ${ }^{2 \dagger}$, S. Das Sarma ${ }^{1,2}$ \\ 1 Joint Quantum Institute, Department of Physics, \\ University of Maryland, College Park, Maryland 20742, USA \\ ${ }^{2}$ Condensed Matter Theory Center, Department of Physics, \\ University of Maryland, College Park, Maryland 20742, USA
}

(Dated: November 21, 2018)

\begin{abstract}
Recent experimental progress in producing ultracold polar molecules with a net electric dipole moment opens up new possibilities to realize novel quantum phases governed by the long-range and anisotropic dipole-dipole interactions. In this work we predict the existence of experimentally observable novel broken-symmetry states with spontaneous interlayer coherence in cold polar molecule bilayers. These exotic states, which are manifestations of collective bilayer quantum entanglement, appear due to strong repulsive interlayer interactions and exhibit properties of superfluids, ferromagnets and excitonic condensates.

PACS numbers: 67.85.-d, 67.85.De, 05.30.Rt, 05.30.Fk
\end{abstract}

During the last decade we have observed the spectacular progress in the realization of various quantum phases using cold atoms. This progress has deepened our understanding of various phenomena such as BCS-BEC crossover of fermions [1] and superfluid-Mott insulator phase transition of bosons in an optical lattice [2]. However, the variety of quantum phases that can be realized in cold atom systems is limited by the short-range nature of the interparticle interactions. Recent progress in producing and manipulating heteronuclear polar molecules [3] provides an opportunity to realize a plethora of novel quantum phases of matter governed by long-range interactions [4. This interesting prospect is made possible by the fact that polar molecules have large electric dipole moments associated with their rotational excitations, which lead to strong, long-range and anisotropic dipole-dipole interactions. The interactions between such polar molecules can be tuned using dc and ac electric fields [1. Below, we concentrate on one intriguing aspect of fermionic polar molecule systems - the possibility to realize bilayer superfluidity (or equivalently bilayer $X Y$ ferromagnetism) with spontaneous interlayer coherence using interlayer molecular repulsion.

Because of the fermionic nature of the molecules even for the spinless fermion case considered here, the interaction has an exchange component in the layer (or pseudospin) index which drives the instability towards the bilayer superfluid phase. The dominant contribution to the exchange energy comes from the short distances (large momenta) where the interlayer dipolar interaction is repulsive. Thus, the novel collective bilayer state we predict arises from a repulsive interaction in sharp contrast to all other superfluid quantum phases discussed in cold atomic fermions where inter-particle attraction leads to

\footnotetext{
*Present address: Microsoft Research, Station Q, Elings Hall, University of California, Santa Barbara, CA 93106, USA

${ }^{\dagger}$ Present address: Department of Physics, College of William and Mary, Williamsburg, VA 23187, USA
}

superfluidity. In the symmetry broken interlayer coherent phase, the particle number in each layer becomes indeterminate in spite of the interlayer single-particle tunneling amplitude being almost zero. Such a state is very analogous to an excitonic superfluid in which excitons formed a quasiparticle in one layer "binding" to a quasihole in the other layer condense into a phase coherent state. Up to this date the clearest evidence for the realization of this type of exciton superfluid state has been observed in semiconductor bilayers in the Quantum Hall $(\mathrm{QH})$ regime, in which the layers are immersed in very high magnetic fields [7]. The unavoidable presence of disorder in solid state systems as well as the nature of the measurement involving finite interlayer tunneling [8] cause complications in the pristine realization of the interlayer superfluid phase in QH bilayers. As a result, vortices and the Berezinskii-Kosterlitz-Thouless (BKT) transition, unambiguous signatures of the interlayer coherent state, have not been observed yet in solid state QH systems. The predicted polar molecule bilayer superfluid phase should be more striking because of the lack of disorder in cold atom systems, the tunability of the interaction strength and the availability of experimental techniques allowing imaging of vortices 9. Moreover, by adding an optical lattice potential in the $x y$-plane it is possible to modify the single-particle dispersion of the particles and model various condensed matter systems. For example, the bilayer cold-polar-molecule system with honeycomb lattice potential will mimic the bilayer graphene system. Thus, the realization of this interesting phase in cold atom systems is of great importance for understanding the instabilities driving the bilayer superfluidity and in general the physics of exciton condensation.

Theoretical model. Our starting point is the Hamiltonian for fermionic polar molecules tightly confined along the $z$-direction by the laser field as shown in Fig. 1 b. We consider two clouds of polar molecules separated by a distance $l_{z}$ much larger than the confinement length $w_{z}$ of the molecules within each layer. When the confinement length $w_{z}$ is much larger than the size of the 

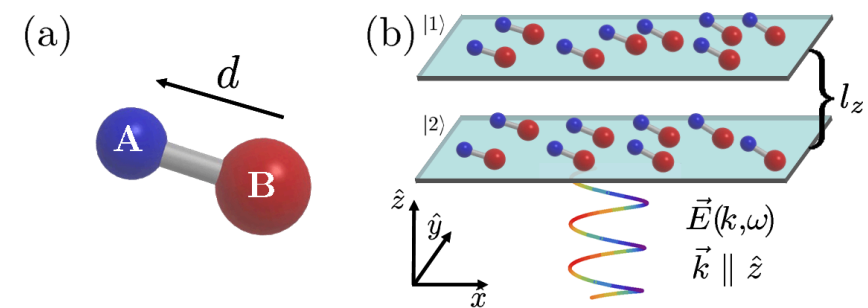

(c)

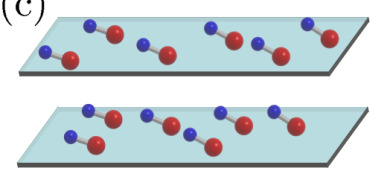

$M=0$ (d)

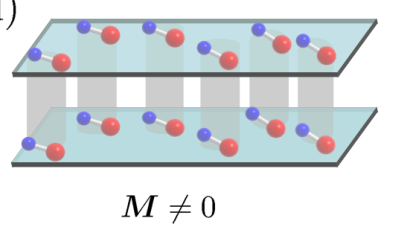

FIG. 1: (Color online) (a) Heteronuclear polar molecules AB with $\mathrm{AB}$ being ${ }^{40} \mathrm{~K}^{87} \mathrm{Rb},{ }^{7} \mathrm{Li}^{40} \mathrm{~K},{ }^{6} \mathrm{Li}^{133} \mathrm{Cs}$. (b) Bilayer system of cold polar molecules in the presence of a circularly polarized ac electromagnetic field propagating along the $z$ direction. Schematic picture of different phases in the bilayers system: normal (c) and pseudospin ferromagnetic (d).

polar molecules, the rotational motion of the molecules is three-dimensional and is described by a $3 \mathrm{D}$ rigid rotor Hamiltonian. Polar molecules have permanent electric dipole moment $d$, which couples to internal rotational degrees of freedom. The dipole moment leads to long-range interlayer and intralayer dipole-dipole interactions. The Hamiltonian of the polar molecules $H$ reads $\left(\hbar=k_{B}=1\right)[1]$

$$
H=\sum_{i}\left(\frac{\boldsymbol{p}_{i}^{2}}{2 m}+B \boldsymbol{J}_{i}^{2}\right)+\sum_{i j} \frac{\boldsymbol{d}_{i} \boldsymbol{d}_{j}-3\left(\boldsymbol{d}_{i} \cdot \hat{\boldsymbol{r}}_{i j}\right)\left(\boldsymbol{d}_{j} \cdot \hat{\boldsymbol{r}}_{i j}\right)}{2 r_{i j}^{3}}
$$

where $\boldsymbol{p}=\left(p_{x}, p_{y}\right)$ is the center-of-mass momentum of a molecule with mass $m, r_{i j}$ is the distance between two molecules, $B$ is the effective rotational energy and $\boldsymbol{J}=\left(J_{x}, J_{y}, J_{z}\right)$ is the angular momentum operator. The rotational eigenstates are $\left|J, M_{J}\right\rangle$ with $J$ and $M_{J}$ denoting the total internal angular momentum and its projection on the quantization axis, respectively.

The orientation of the dipole moments can be controlled with dc and ac electric fields Fig. 1p, 1]. The transition dipole moment between the states with $J=0$ and $J=1$ is $d_{t} \equiv\left|\left\langle 0,0|\boldsymbol{d}| 1, M_{J}\right\rangle\right|=d / \sqrt{3}$ with $M_{J}=0, \pm 1$. A circularly polarized ac electric field $\boldsymbol{E}_{\mathrm{ac}}(t)$ propagating along $z$-direction, see Fig. 1 $\mathrm{b}$, drives transitions between the rotational states $|0,0\rangle$ and $|1,1\rangle$ with Rabi frequency $\Omega_{R}=d_{t} E_{\mathrm{ac}}$. If the frequency of the field $\omega$ is close to the transition frequency $\omega_{0}=2 B$ between the states $|0,0\rangle$ and $|1,1\rangle$ (i.e. the detuning $\Delta=\omega-\omega_{0} \ll \omega_{0}$ ), the leading effect of the electric field is to mix these two states. Within the rotating wave approximation, the dressed states are given by $| \pm\rangle=\alpha_{ \pm}|0,0\rangle \pm \alpha_{\mp} e^{-i \omega t}|1,1\rangle$, where $\alpha_{+}=-\Gamma / \sqrt{\Gamma^{2}+\Omega_{R}^{2}}, \alpha_{-}=\Omega_{R} / \sqrt{\Gamma^{2}+\Omega_{R}^{2}}$ and $2 \Gamma=\Delta+\sqrt{\Delta^{2}+4 \Omega_{R}^{2}}$ [1, 4]. Polar molecules can be prepared in the internal state $|+\rangle_{i}$ by an adiabatic switching

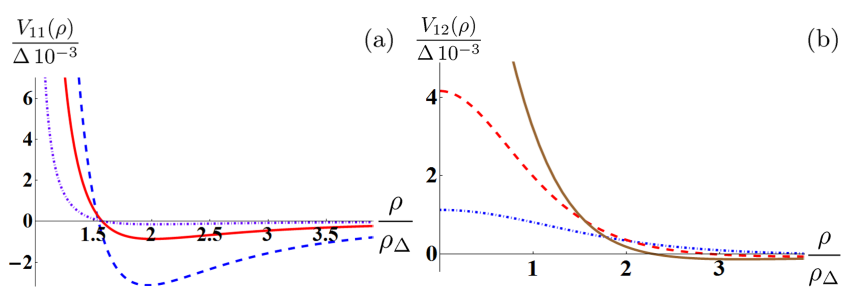

FIG. 2: (Color online) (a) Intralayer Born-Oppenheimer potential for polar molecules. The dashed(blue), solid(red) and dot-dashed(violet) lines correspond to $\Omega_{R} / \Delta=1 / 4$, $\Omega_{R} / \Delta=1 / 8$ and $\Omega_{R} / \Delta=1 / 20$, respectively. (b) Interlayer Born-Oppenheimer potential for $\Omega_{R} / \Delta=1 / 8$. The dash$\operatorname{dot}($ blue), solid(red) and dashed(brown) lines correspond to $l_{z} / \rho_{\Delta}=3,2,1.5, l_{z} / \rho_{\Delta}=1.5$, respectively. For typical interparticle distances considered here the interlayer interaction is repulsive. Here $\Omega_{R} / \Delta=1 / 8$ and $l_{z} / \rho_{\Delta}=3$.

of the microwave field. In this case, the effective interaction between polar molecules $V_{\text {eff }}(r)$ is given by the dressed Born-Oppenheimer potential adiabatically connected to the state $|+\rangle_{i} \otimes|+\rangle_{j}$, see Fig.1 of the supplementary material. At large distances the dipolar interaction can be obtained perturbatively by first calculating the effective dipole moment $\langle+|\boldsymbol{d}|+\rangle=d_{\text {eff }}(\cos \omega t, \sin \omega t, 0)$ with $d_{\text {eff }}=-\sqrt{2} \alpha_{+} \alpha_{-} d_{t}$. The time-averaged interaction between dipoles in layers $\lambda$ and $\lambda^{\prime}$ takes the form

$$
V_{\mathrm{eff}}^{\lambda \lambda^{\prime}}(\rho)=d_{\mathrm{eff}}^{2}\left(\frac{1}{\left(z_{\lambda \lambda^{\prime}}^{2}+\rho^{2}\right)^{\frac{3}{2}}}-\frac{3}{2} \frac{\rho^{2}}{\left(z_{\lambda \lambda^{\prime}}^{2}+\rho^{2}\right)^{\frac{5}{2}}}\right),
$$

where $\boldsymbol{\rho}=(x, y)$ is the $2 \mathrm{D}$ coordinate, and $z_{\lambda \lambda^{\prime}}=l_{z}$ for $\lambda \neq \lambda^{\prime}$ and zero otherwise. At short distances, when the dipolar interaction energy is comparable with the detuning, the above perturbative treatment breaks down. In order to find the Born-Oppenheimer potential at short distances $\rho \leq \rho_{\Delta} \equiv\left(d_{t}^{2} / \Delta\right)^{\frac{1}{3}}$, it is necessary to account for all couplings between different angular momentum channels within the $J=0,1$ manifold [1]. The exact Born-Oppenheimer potentials are shown in Fig. 2. One can notice that the effective intralayer dipole-dipole interaction between polar molecules prepared in the state $|+\rangle$ becomes repulsive at $\rho \sim \rho_{\Delta}$ due to the presence of avoided crossings with other field-dressed levels 4 . For ${ }^{6} \mathrm{Li}^{133} \mathrm{Cs}$ molecules, typical parameters are $d \approx 6.3 \mathrm{D}$, $B \approx 6 \mathrm{GHz}$, and $\Delta \approx 10 \mathrm{MHz}$ yielding the length scale $\rho_{\Delta} \approx 50 \mathrm{~nm}$ that is much larger than the characteristic scale of dipole-dipole interactions $\rho_{B}=\left(d^{2} / B\right)^{1 / 3} \sim 1 \mathrm{~nm}$, which sets the short-range cutoff. Thus, for typical densities of polar molecules considered here, $n_{0} \sim 10^{7} \mathrm{~cm}^{-2}$, the ac electric field shields the molecules from short-range inelastic collisions and prevents from the collapse of the system [1, 4, 10], see supplementary material.

Henceforth, we consider the dilute gases of polar molecules, where the interparticle distance is larger than $\rho_{\Delta}$, i.e. $l_{z}, n_{0}^{-1 / 2} \gg \rho_{\Delta}$, and the interaction between particles is given by the dressed Born-Oppenheimer po- 
tentials shown in Fig. 2, In order to avoid unwanted inelastic collisions leading to the decay of the molecules in an s-wave channel, we assume the molecules to be spinpolarized. In this limit, the effective second-quantized Hamiltonian of the bilayer system takes the form

$$
\begin{aligned}
H & =\sum_{k \lambda}\left(\varepsilon(k)-\mu_{\lambda}\right) c_{k \lambda}^{\dagger} c_{k \lambda} \\
& +\frac{1}{2} \sum_{q, k, k^{\prime}, \lambda \lambda^{\prime}} V_{\mathrm{eff}}^{\lambda \lambda^{\prime}}(q) c_{k+q \lambda}^{\dagger} c_{k^{\prime}-q \lambda^{\prime}}^{\dagger} c_{k^{\prime} \lambda^{\prime}} c_{k \lambda},
\end{aligned}
$$

where $c_{k \lambda}$ and $c_{k \lambda}^{\dagger}$ are the fermion creation and annihilation operators for a molecule with momentum $\boldsymbol{k}$ in layer $\lambda$. The strength of the dipolar interactions can be characterized by the dimensionless parameter $r_{s}=$ $d_{\text {eff }}^{2} m \sqrt{n_{0}} / 2 \pi$. As $r_{s}$ is increased, the bilayer system becomes susceptible to various instabilities driven by the dipolar interactions. Here we concentrate on the instabilities induced by the interlayer interactions and neglect the instabilities induced by the intralayer interactions, see supplementary material for the justification of such approximations.

To understand the interlayer instability, it is convenient to draw an analogy with ferromagnetism and introduce pseudospin- $1 / 2$ operators $2 \hat{\boldsymbol{m}}_{i}=c_{i \lambda}^{\dagger} \boldsymbol{\sigma}_{\lambda \lambda^{\prime}} c_{i \lambda^{\prime}}$. The spinors $|\uparrow\rangle$ and $|\downarrow\rangle$ represent the states in which molecules are in layer 1 or 2 , respectively. When $l_{z} \gg k_{F}^{-1}$, with $k_{F}=\sqrt{4 \pi n_{0}}$ being the Fermi momentum, the molecules in different layers are uncorrelated, and the many-body state of the system is given by

$$
\left|\Psi_{\mathrm{N}}\right\rangle=\prod_{k \leq k_{F}} c_{k 1}^{\dagger} \prod_{k^{\prime} \leq k_{F}} c_{k^{\prime} 2}^{\dagger}|0\rangle .
$$

For equal densities in the layers $n_{1}=n_{2}=n_{0}$, the total magnetization $\boldsymbol{M}$ is zero, $\boldsymbol{M}=\left\langle\Psi_{\mathrm{N}}\left|\sum_{i} \hat{\boldsymbol{m}}_{i}\right| \Psi_{\mathrm{N}}\right\rangle=0$, similar to paramagnets. The normal state $\left|\Psi_{\mathrm{N}}\right\rangle$ minimizes the kinetic energy at the expense of the potential energy, which is at its maximum. When the interlayer distance becomes smaller than $k_{F}^{-1}$, the potential energy becomes large and at some point starts to dominate over the kinetic energy. In this case, the system favors the state in which fermions in different layers are correlated in a way that minimizes the interaction energy, i.e. the molecule in layer 1 is coupled to a "hole" in layer 2. At the mean-field level such correlations are captured by an order parameter $\Delta_{12} \propto\left\langle c_{k 1}^{\dagger} c_{k 2}\right\rangle \neq 0$. Since the product wavefunction $\Psi_{\mathrm{N}}$ does not have such entanglement between the layers, the bilayer system should undergo a quantum phase transition as a function of the distance $l_{z}$ or the strength of the dipole moment $d_{\text {eff }}$. The many-body wavefunction minimizing the interaction energy takes the form

$$
\left|\Psi_{\mathrm{FM}}\right\rangle=\prod_{k \leq \sqrt{2} k_{F}}\left(\frac{c_{k 1}^{\dagger}+e^{i \varphi} c_{k 2}^{\dagger}}{\sqrt{2}}\right)|0\rangle .
$$

In this entangled state the state of the molecule is given by the coherent superposition of the amplitudes in different layers. Thus, even in the absence of tunneling, the molecule layer index becomes uncertain. Using the spin analogy, the state $\left|\Psi_{\mathrm{FM}}\right\rangle$ has non-zero magnetization $\boldsymbol{M}=\left\langle\Psi_{\mathrm{FM}}\left|\sum_{i} \hat{\boldsymbol{m}}_{i}\right| \Psi_{\mathrm{FM}}\right\rangle \neq 0$ with $\boldsymbol{M}$ lying in the $x y$-plane. Similar to superconductors, this ferromagnetic state $\left|\Psi_{\mathrm{FM}}\right\rangle$ spontaneously breaks $U(1)$ symmetry and develops interlayer coherence. In this state the phase difference between different layers $\varphi$ is well defined and the number of molecules in each layer fluctuates satisfying the uncertainty relations $\Delta m_{z} \Delta \varphi \geq 1 / 2$ [11] despite the absence of interlayer tunneling in Eq. (3).

The phase diagram between the two competing states - normal $\left|\Psi_{\mathrm{N}}\right\rangle$ and pseudospin ferromagnetic $\left|\Psi_{\mathrm{FM}}\right\rangle$ - can be obtained using variational mean-field calculation [12]. The total energy per area $\mathcal{A}$ of the bilayer system in the normal phase is

$$
\frac{E_{\mathrm{N}}}{\mathcal{A}}=\frac{2 \pi n_{0}^{2}}{m}\left(1+r_{s} \frac{Z\left(2 k_{F} \rho_{\Delta}\right)}{k_{F} \rho_{\Delta} \frac{d_{\mathrm{eff}}^{2}}{d_{t}^{2}}}\right),
$$

where the dimensionless function $Z(a)$, which describes intralayer interaction, is defined as

$$
Z(a)=\frac{32}{\sqrt{\pi}} \int_{0}^{1} x d x f(x) \frac{\left[V_{\mathrm{eff}}^{(11)}(0)-V_{\mathrm{eff}}^{(11)}(a x)\right]}{\Delta \rho_{\Delta}^{2}}
$$

with $f(x)=\arccos (x)-x \sqrt{1-x^{2}}$. The momentum dependence of the intralayer interaction potential $V_{\text {eff }}^{(11)}(q)$ is shown in Fig. 1 (b) of the supplementary material. One can notice that the contribution of the interlayer Hartree term is zero here because $V_{\mathrm{eff}}^{12}(q)=d_{\mathrm{eff}}^{2} \pi q e^{-q l_{z}}$ goes to zero as $q \rightarrow 0$. To calculate the energy of the system in the interlayer coherent state $\left|\Psi_{\mathrm{FM}}\right\rangle$ we first introduce the order parameter

$$
\Delta_{12}(k)=\frac{1}{2} \sum_{q} V_{12}(q) e^{-i \varphi}\left\langle c_{1}^{\dagger}(k+q) c_{2}(k+q)\right\rangle,
$$

which takes into account interlayer correlations. The order parameter is obtained by numerically solving the selfconsistent equation above, subject to the total particle number conservation constraint. Because of the dipolar nature of the interaction $\Delta_{12}(k)$ has momentum dispersion. The dependence of $\Delta_{12}(0)$ on $d_{\text {eff }}$ and $l_{z}$ obtained self-consistently is shown in Fig. 3 (a). At the mean-field level, the Hamiltonian (3) can be diagonalized using a Bogoliubov transformation yielding the many-body variational wavefunction (5). For sufficiently large interactions the lowest energy state of the system corresponds to the pseudospin ferromagnetic state fully polarized in the $x y$-plane [12, see also supplementary material. The total energy of the system per area in the interlayer coherent state is given by

$$
\frac{E_{\mathrm{FM}}}{\mathcal{A}}=\mathcal{E}_{0}\left[1-r_{s} F\left(\sqrt{8} k_{\mathrm{F}} l_{z}\right)+\frac{r_{s}}{2} \frac{Z\left(\sqrt{8} k_{F} \rho_{\Delta}\right)}{k_{F} \rho_{\Delta} \frac{d_{\mathrm{eff}}^{2}}{d_{t}^{2}}}\right],
$$



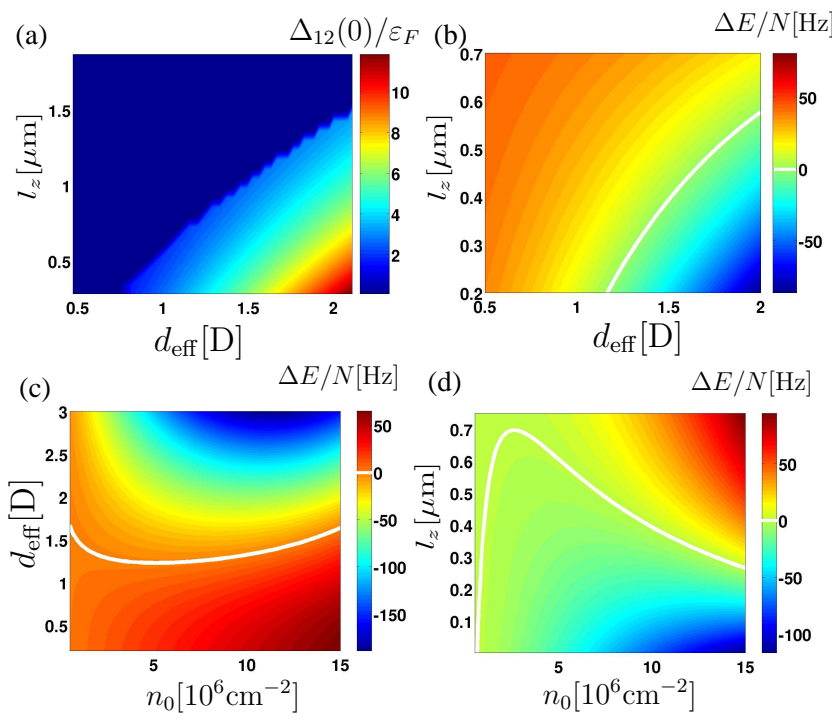

FIG. 3: (Color online) The dependence of $\Delta_{12}(0)$ and $\Delta E$ on $l_{z}$ and $d_{\text {eff }}$ is shown in (a) and (b), respectively. Here we used $\rho_{\Delta}=50 \mathrm{~nm}, n_{0}=10^{7} \mathrm{~cm}^{-2}$ and $m=139 m_{p}$ with $m_{p}$ being the proton mass. The dependence of $\Delta E$ on $n_{0}$ and $d_{\text {eff }}$ at fixed $l_{z}=0.3 \mu \mathrm{m}$ and on $n_{0}$ and $l_{z}$ at fixed $d_{\text {eff }}=1.5 \mathrm{D}$ is plotted in (c) and (d), respectively.

where $\mathcal{E}_{0}=4 \pi n_{0}^{2} / m$ and the function $F(a)$ describing the interlayer exchange contribution reads

$$
F(a)=32 \sqrt{2 \pi} \int_{0}^{1} x^{2} d x\left(\arccos (x)-x \sqrt{1-x^{2}}\right) e^{-a x} .
$$

The energy difference between normal and ferromagnetic phase $\Delta E / \mathcal{A}=\left(E_{\mathrm{FM}}-E_{\mathrm{N}}\right) / \mathcal{A}$ determines the mean-field phase diagram for the bilayers of polar molecules shown in Fig. 3 .

The long wavelength Hamiltonian describing the phase fluctuations is

$$
H=\frac{1}{2} \int d^{2} \boldsymbol{r} \rho_{s}|\nabla \varphi|^{2}
$$

where $\rho_{s}$ is the "spin stiffness", which is the result of the loss of interaction energy due to the spatial variations of order parameter phase $\varphi$. The effective $X Y$ model defined by Eq. (11) undergoes BKT transition associated with unbinding of vortex pairs at the temperature $T_{\mathrm{BKT}} \approx \pi \rho_{s} / 2$. The "spin stiffness" can be calculated within linear response theory, which yields the result $\rho_{s}=n_{0} / 2 m$, similar to the one in superfluids. Thus, the BKT transition in the bilayer system of polar molecules occurs at the temperature $T_{\mathrm{BKT}} \approx \varepsilon_{F} / 8$ with $\varepsilon_{F}=k_{F}^{2} / 2 m$, that for $n_{0} \sim 10^{7} \mathrm{~cm}^{-2}$ corresponds to a temperature that should be accessible in the near future [13]. In the pseudospin ferromagnetic phase vortices are correlated in different layers, and the BKT transition in the bilayer system can be detected by imaging vortices using matter wave heterodyning techniques [9].

In summary, we predict an unusual broken-symmetry phase with spontaneous interlayer coherence in a bilayer system of cold polar molecules. Our main findings, summarized in the phase diagram shown in Fig. 3, indicate that the experimental observation of such phase requires low densities of cold polar molecules $n_{0} \sim 10^{7} \mathrm{~cm}^{-2}$, realistic dipole moments $d_{\text {eff }} \sim 1 \mathrm{D}$, and reasonably low temperatures $T \sim 1 \mathrm{nK}$. Given that for these parameters the inelastic decay rate is small [4, the detection of this exotic many-body state should be within the experimental reach in the near future. The novel phase we predict is an interlayer entangled state, arising from the repulsive part of the dipolar interaction and exhibiting superfluidity (or, equivalently, $X Y$ pseudospin ferromagnetism) between the layers rather than within the layers.

Acknowledgements. We thank P. S. Julienne, I. Spielman and T. Porto, and most particularly D.-W. Wang, for helpful discussions. This work is supported by USAFOSR-MURI and NSF-JQI-PFC.
[1] M. Greiner, C. Regal, and D. Jin, Nature 426, 537 (2003); C. Chin et al., Science 305, 1128 (2004); M. Zwierlein et al., Nature 435, 1047 (2005).

[2] M. Greiner et al., Nature 415, 39 (2002).

[3] K. K. Ni et al., Science 322, 231 (2008); J. Deiglmayr et al., Phys. Rev. Lett. 101, 133004 (2008); F. Lang et al., Phys. Rev. Lett. 101, 133005 (2008); S. Ospelkaus et al., Nat. Phys. 4, 622 (2008).

[4] A. Micheli, G. K. Brennen, and P. Zoller, Nat. Phys. 5, 341 (2006); D.-W. Wang, M. D. Lukin, and E. Demler, Phys. Rev. Lett. 97, 180413 (2006); D.-W. Wang, Phys. Rev. Lett. 98, 060403 (2007); H. P. Büchler et al., Phys. Rev. Lett. 98, 060404 (2007); C.-M. Chang et al., Phys. Rev. A 79, 053630 (2009).
[4] N. R. Cooper and G. V. Shlyapnikov, Phys. Rev. Lett. 103, 155302 (2009).

[1] A. Micheli et al., Phys. Rev. A 76, 043604 (2007); A. V. Gorshkov et al., Phys. Rev. Lett. 101, 073201 (2008).

[7] J. Eisenstein and A. MacDonald, Nature 432, 691 (2004).

[8] I. B. Spielman et al., Phys. Rev. Lett. 84, 5808 (2000).

[9] Z. Hadzibabic et al. , Nature 441, 1118 (2006).

[10] R. Napolitano, J. Weiner, and P. S. Julienne, Phys. Rev. A 55, 1191 (1997).

[11] K. Moon et al., Phys. Rev. B 51, 5138 (1995).

[12] L. Zheng, M. W. Ortalano, and S. Das Sarma, Phys. Rev. B 55, 4506 (1997).

[13] J. Ye (2009), private communication. 


\section{SUPPLEMENTARY INFORMATION}

In this document we elaborate on the analysis of the various instabilities in the bilayer system of cold polar molecules. We begin with the discussion of the role of the intralayer interactions in stabilizing the cold-polarmolecule Fermi gas.

\section{A. Stability analysis of the single layer}

The Hamiltonian for a single layer 2D Fermi gas of cold polar molecules reads

$$
H=\sum_{k}(\varepsilon(k)-\mu) c_{k \lambda}^{\dagger} c_{k}+\frac{1}{2} \sum_{q, k, k^{\prime}} V_{\mathrm{eff}}^{(11)}(q) c_{k+q}^{\dagger} c_{k^{\prime}-q}^{\dagger} c_{k^{\prime}} c_{k}
$$

The intralayer interaction is given by the dressed BornOppenheimer potential $V_{11}(\rho)$ [1, 2], which has a shortdistance repulsive core and an attractive tail at large distances as shown in Fig. 5a of the main text. The crossover between these two regimes occurs at the length scale $\rho_{\Delta}$, which is determined by the detuning $\Delta$, see Fig. 4. Thus, by changing the detuning frequency one can control the strength of the intralayer interaction.

The energy of the single layer in the Hartree-Fock approximation [3] is given by

$$
\begin{aligned}
\frac{E_{\mathrm{t}}^{(\mathrm{sl})}}{\mathcal{A}} & =\sum_{k} \frac{k^{2}}{2 m} n_{k}+\frac{1}{2} \sum_{k, q}\left[V_{\mathrm{eff}}^{(11)}(0)-V_{\mathrm{eff}}^{(11)}(q)\right] n_{k} n_{k+q} \\
& =\frac{\pi n_{0}^{2}}{m}+\frac{1}{2} \int \frac{d^{2} \boldsymbol{q}}{(2 \pi)^{2}}\left[V_{\mathrm{eff}}^{(11)}(0)-V_{\mathrm{eff}}^{(11)}(q)\right] I\left(q, k_{F}\right),
\end{aligned}
$$

where $n_{k}=\Theta\left(k_{F}-k\right)$ with $\Theta(x)$ being the Heaviside step function. The dimensionless function $I(q, x)$ is given by

$$
\begin{aligned}
I(q, x) & =\int \frac{d^{2} \boldsymbol{k}}{(2 \pi)^{2}} \Theta\left(x-\sqrt{k^{2}+q^{2}+2 \boldsymbol{k q}}\right) \Theta(x-k) \\
& =\frac{x^{2}}{2 \pi^{2}} \Theta(2 x-q)\left[\arccos \left(\frac{q}{2 x}\right)-\left(\frac{q}{2 x}\right) \sqrt{1-\left(\frac{q}{2 x}\right)^{2}}\right],
\end{aligned}
$$

and the momentum dependence of the intralayer potential $V_{\mathrm{eff}}^{(\lambda \lambda)}(0)-V_{\mathrm{eff}}^{(\lambda \lambda)}(q)$ is shown in Fig. 5b. Then, the total energy of the system becomes

$$
\begin{aligned}
\frac{E_{\mathrm{t}}^{(\mathrm{sl})}}{\mathcal{A}} & =\frac{\pi n_{0}^{2}}{m}+\frac{8}{\pi} n_{0}^{2} \int_{0}^{1} x d x\left[V_{\mathrm{eff}}^{(11)}(0)-V_{\mathrm{eff}}^{(11)}\left(2 k_{F} \rho_{\Delta} x\right)\right] f(x) \\
& =\frac{\pi n_{0}^{2}}{m}\left(1+r_{s} \frac{Z\left(2 k_{F} \rho_{\Delta}\right)}{k_{F} \rho_{\Delta} \frac{d_{\mathrm{eff}}^{2}}{d_{t}^{2}}}\right)
\end{aligned}
$$

with $r_{s}=d_{\text {eff }}^{2} m \sqrt{n_{0}} / 2 \pi$. The dimensionless function $Z(a)$ is defined as

$$
Z(a)=\frac{32}{\sqrt{\pi}} \int_{0}^{1} f(x) x d x \frac{\left[V_{\mathrm{eff}}^{(11)}(0)-V_{\mathrm{eff}}^{(11)}(a x)\right]}{\Delta \rho_{\Delta}^{2}},
$$

!hb

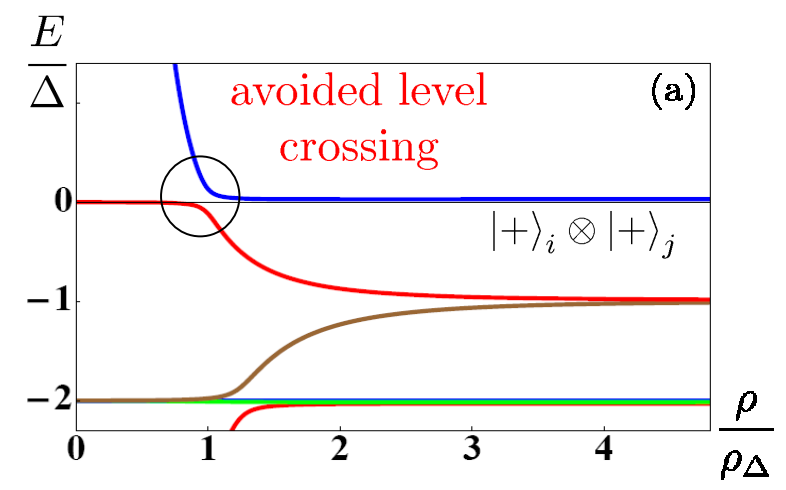

FIG. 4: Two-body energy levels as a function of the distance for $\Omega_{R} / \Delta=1 / 8$.

where $f(x)=\arccos (x)-x \sqrt{1-x^{2}}$. Since the integral 16 involves both the contribution from the attractive tail and repulsive core of the potential $V_{\mathrm{eff}}^{(11)}(\rho)$ (see Fig 5), the intralayer interaction energy depends on the parameter $k_{F} \rho_{\Delta}$. For typical densities considered here $n_{0} \approx 10^{7} \mathrm{~cm}^{-2}$ and $\rho_{\Delta} \approx 50 \mathrm{~nm}$ (i.e. $k_{F} \rho_{\Delta} \approx 0.06$ ), the compressibility of the system $\kappa^{-1}=\frac{n_{0}^{2}}{\mathcal{A}} \frac{\partial^{2} E_{\mathrm{t}}^{(\mathrm{sl})}}{\partial n_{0}^{2}}$ is positive, see Fig 5, and thus, the ac electric field allows one to stabilize the Fermi gas and prevent phase separation.

\section{B. Intralayer superfluid instability.}

We now discuss the possibility of the superfluid instability of the Fermi surface in a single layer of cold polar molecules. It was shown in Ref. 4] that the intralayer interaction can lead to p-wave pairing. The weak coupling BCS theory developed in Ref. [4] is valid for $r_{s} \ll 1$, and the evaluation of the superfluid $T_{c}$ for typical parameters considered here $\left(r_{s} \lesssim 1\right)$ requires developing a strong coupling theory of superfluidity, which is beyond the scope of the present paper. However, we note that by tuning the ratio $\Omega_{R} / \Delta$ the depth of the attractive potential can be decreased as shown in Fig. 5 a indicating that it is possible to substantially suppress the intralayer superfluid $T_{c}$ or eliminate such instability altogether. To demonstrate this, we calculate the intralayer interaction in the p-wave channel $V^{(l=1)}$

$$
V^{(l)} \equiv V^{(l)}\left(k_{F}, k_{F}\right)=2 \pi \int_{0}^{\infty} \rho d \rho J_{l}^{2}\left(k_{F} \rho\right) V^{(11)}(\rho),
$$

which can be controlled by changing $\Omega_{R} / \Delta$. Here $J_{l}\left(k_{F} \rho\right)$ is the Bessel function of order $l$. Due to the short range repulsion the potential $V^{(l=1)}$ changes sign as a function of $k_{F} \rho_{\Delta}$, see Fig. $5 \mathrm{~d}$. For the relevant parameters considered here $\Omega_{R} / \Delta=1 / 8$ and $k_{F} \rho_{\Delta} \approx 0.06$, the potential $V^{(l=1)}$ is positive, and the analysis of the 


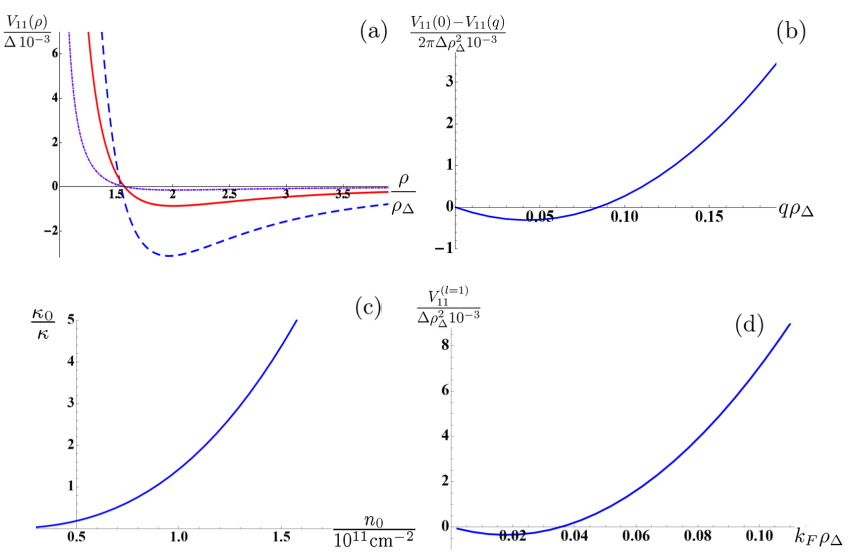

FIG. 5: (a) Intralayer Born-Oppenheimer potential for polar molecules. The dashed(blue), solid(red) and dotdashed(violet) lines correspond to different Rabi frequencies: $\Omega_{R} / \Delta=1 / 4, \Omega_{R} / \Delta=1 / 8$ and $\Omega_{R} / \Delta=1 / 20$, respectively. (b) Fourier transform of the intralayer Born-Oppenheimer potential for $\Omega_{R} / \Delta=1 / 8$. (c) The compressibility of the singlelayer cold polar Fermi gas as a function of density $n_{0}$. Here $\kappa_{0}=m / \pi$. (d) The dependence of the p-wave potential $V^{(l=1)}$ on the dimensionless parameter $k_{F} \rho_{\Delta}$.

spontaneous bilayer coherence neglecting the intralayer instabilities in the particle-particle channel is justified. We note here that there is no inconsistency with the conclusion of Cooper and Shlyapnikov [4] since for the parameters used in Ref. $4 .\left(\Omega_{R} / \Delta=1 / 4\right.$ and $\left.\rho_{\Delta}=30 \mathrm{~nm}\right)$ the potential is $V^{(l=1)}$ is indeed negative leading to the p-wave superfluidity.

\section{Effect of intralayer interactions on the pseudo-spin instabilities}

In this section we provide details on the calculation of the energy of the bilayer system of cold polar molecules within variational mean-field theory. We begin by analyzing the total energy of the system per area in the normal phase described by the wavefunction $\left|\Psi_{\mathrm{N}}\right\rangle$ (see Eq. (4) of the main text):

$\frac{E_{\mathrm{N}}}{\mathcal{A}}=\frac{2 \pi n_{0}^{2}}{m}+\frac{16}{\pi} n_{0}^{2} \int_{0}^{1} x d x\left[V_{\mathrm{eff}}^{(11)}(0)-V_{\mathrm{eff}}^{(11)}\left(2 k_{F} \rho_{\Delta} x\right)\right] f(x)$, $=\frac{2 \pi n_{0}^{2}}{m}\left(1+r_{s} \frac{Z\left(2 k_{F} \rho_{\Delta}\right)}{k_{F} \rho_{\Delta} \frac{d_{\mathrm{eff}}^{2}}{d_{t}^{2}}}\right)$,

The energy $E_{\mathrm{N}}$ essentially follows from Eq. 15 multiplied by the number of layers.

We now discuss the energy of the bilayer system in the superfluid state. Assuming that the dominant contribution to the energy comes from interlayer interactions, we introduce the mean field $\Delta_{12}(k)$ (see Eq. (8) of the main text) and neglect for now the intralayer interactions. At the mean-field level, the Hamiltonian

$$
H_{\mathrm{MF}}=\sum_{k \lambda}\left(\frac{k^{2}}{2 m}-\mu\right) c_{k \lambda}^{\dagger} c_{k \lambda}-\sum_{k}\left[\Delta_{12}(k) c_{2}^{\dagger}(k) c_{1}(k)+h . c .\right]
$$

can be diagonalized via canonical transformation yielding the energy spectrum $\varepsilon_{ \pm}(k)=\left(\frac{k^{2}}{2 m}-\mu\right) \pm\left|\Delta_{12}(k)\right|$. Assuming that all molecules occupy the lowest energy band $\varepsilon_{-}(k)$, the wavefunction corresponding to such state $\left|\Psi_{\mathrm{FM}}\right\rangle$ is given by Eq. (5) of the main text. We calculate the order parameter self-consistently by enforcing the above constraint. Using the variational wavefunction $\left|\Psi_{\mathrm{FM}}\right\rangle$, we then calculate the total energy of the system. The contribution of the intralayer interaction reads

$$
\begin{aligned}
\frac{E_{\text {int }}^{(\lambda \lambda)}}{\mathcal{A}} & =\frac{1}{8} \sum_{k, q}\left[V_{\mathrm{eff}}^{(\lambda \lambda)}(0)-V_{\mathrm{eff}}^{(\lambda \lambda)}(q)\right] n_{k \lambda} n_{k+q \lambda} \\
& =\frac{1}{8} \int \frac{d^{2} \boldsymbol{q}}{(2 \pi)^{2}}\left[V_{\mathrm{eff}}^{(\lambda \lambda)}(0)-V_{\mathrm{eff}}^{(\lambda \lambda)}(q)\right] I\left(q, \sqrt{2} k_{F}\right)
\end{aligned}
$$

and the total energy in the interlayer-coherent superfluid phase is given by

$$
\begin{aligned}
\frac{E_{\mathrm{FM}}}{\mathcal{A}} & =\frac{4 \pi n_{0}^{2}}{m}\left[1-r_{s} F\left(\sqrt{8} k_{F} l_{z}\right)\right] \\
& +\frac{16}{\pi} n_{0}^{2} \int_{0}^{1} x d x\left[V_{\mathrm{eff}}^{(11)}(0)-V_{\mathrm{eff}}^{(11)}\left(\sqrt{8} k_{F} \rho_{\Delta} x\right)\right] f(x) \\
& =\frac{4 \pi n_{0}^{2}}{m}\left[1-r_{s} F\left(\sqrt{8} k_{F} l_{z}\right)+\frac{r_{s}}{2} \frac{Z\left(\sqrt{8} k_{F} \rho_{\Delta}\right)}{k_{F} \rho_{\Delta} \frac{d_{\mathrm{eff}}^{2}}{d_{t}^{2}}}\right] .
\end{aligned}
$$

Here the function $F(a)$ is defined as

$$
\begin{aligned}
F(a) & =32 \sqrt{2 \pi} \int_{0}^{1} x^{2} d x\left(\arccos (x)-x \sqrt{1-x^{2}}\right) e^{-a x} \\
& =\frac{32 \sqrt{2 \pi}}{3 a^{4}}\left[a\left(3 \pi-4 a+15 \pi I_{0}(a)\right)-3 \pi\left(12+a^{2}\right) I_{1}(a)\right. \\
& \left.+3 \pi\left(2+a^{2}\right) L_{1}(a)-15 \pi a L_{2}(a)\right]
\end{aligned}
$$

with $I_{n}(x)$ and $L_{n}(x)$ being the modified Bessel and Struve functions, respectively. As follows from Eq.211, the magnitude of the intralayer and interlayer interactions depend on different physical parameters $k_{F} l_{z}$ and $k_{F} \rho_{\Delta}$. Thus, one can control their relative contributions to the total energy so that the isospin ferromagnetic state is favorable.

We conclude this section by calculating the energy of the system in the Ising ferromagnetic state described by the wavefunction $\Psi_{\mathrm{IM}}=\prod_{k<\sqrt{2} k_{F}} c_{1 k}^{\dagger}|0\rangle$. Such state has the pseudospin magnetization $\boldsymbol{M}$ aligned along the $z$ axis, which corresponds to all molecules being in a single layer. The intralayer interaction energy in this state can 
be written as

$$
\begin{aligned}
\frac{E_{\mathrm{int}}^{(\mathrm{IM})}}{\mathcal{A}} & =\frac{1}{2} \sum_{k, q}\left[V_{\mathrm{eff}}^{(11)}(0)-V_{\mathrm{eff}}^{(11)}(q)\right] n_{k 1} n_{k+q 1} \\
& =\frac{1}{2} \int \frac{d^{2} \boldsymbol{q}}{(2 \pi)^{2}}\left[V_{\mathrm{eff}}^{(\lambda \lambda)}(0)-V_{\mathrm{eff}}^{(\lambda \lambda)}(q)\right] I\left(q, \sqrt{2} k_{F}\right) \\
& =\frac{32}{\pi} n_{0}^{2} \int_{0}^{1} x d x\left[V_{\mathrm{eff}}^{(11)}(0)-V_{\mathrm{eff}}^{(11)}\left(\sqrt{8} k_{F} \rho_{\Delta} x\right)\right] f(x) .
\end{aligned}
$$

The total energy of the system in the Ising ferromagnetic state reads

$$
\begin{aligned}
\frac{E_{\mathrm{t}}^{(\mathrm{IM})}}{\mathcal{A}} & =\frac{4 \pi n_{0}^{2}}{m}+\frac{32}{\pi} n_{0}^{2} \int_{0}^{1} x d x\left[V_{\mathrm{eff}}^{(11)}(0)-V_{\mathrm{eff}}^{(11)}\left(\sqrt{8} k_{F} \rho_{\Delta} x\right)\right] f(x) \\
& =\frac{4 \pi n_{0}^{2}}{m}\left(1+r_{s} \frac{Z\left(\sqrt{8} k_{F} \rho_{\Delta}\right)}{k_{F} \rho_{\Delta} \frac{d_{\mathrm{eff}}^{2}}{d_{t}^{2}}}\right) .
\end{aligned}
$$

[1] A. Micheli et al., Phys. Rev. A 76, 043604 (2007);

[2] A. V. Gorshkov et al., Phys. Rev. Lett. 101, 073201 (2008).

[3] C.-K. Chan, C. Wu, W.-C. Lee, and S. Das Sarma, Phys. Rev. A 81, 023602 (2010)
For typical parameters considered here, the Ising pseudospin ferromagnetic state $\left|\Psi_{\mathrm{IM}}\right\rangle$ has higher energy than the state $\left|\Psi_{\mathrm{FM}}\right\rangle$, and thus, can be ignored.
[4] N. R. Cooper and G. V. Shlyapnikov, Phys. Rev. Lett. 103, 155302 (2009). 